\title{
Pinos transcorticais e gesso associados à aplicação local de plasma rico em plaquetas no tratamento de fratura do III metatarsiano em potro
}

\author{
Transfixation pin cast associated with local platelet rich plasma for treatment of third metatarsal \\ fracture in a filly
}

\author{
Marcos Jun Watanabe ${ }^{I^{*}}$ Ana Lúcia Milluzi Yamada ${ }^{\mathrm{I}}$ Ana Liz Garcia Alves ${ }^{\mathrm{I}}$ \\ Juliana de Moura Alonso ${ }^{\mathrm{I}}$ Roberta Galvano Barbosa ${ }^{\text {II }}$ Cristina de Faria Mantovani ${ }^{\mathrm{I}}$ \\ Carlos Alberto Hussni ${ }^{I}$
}

- NOTA -

RESUMO

\begin{abstract}
Em potros até um ano de idade, as fraturas do III osso metacarpiano (McIII) ou III metatarsiano (MtIII) são principalmente atribuidas ao trauma. A redução aberta e a imobilização do foco com implantes constituem o tratamento cirúrgico recomendado. Uma potra com três meses de idade e $150 \mathrm{~kg}$, da raça Quarto de Milha, que apresentava umafratura diafisária multifragmentar em cunha do MtIII direito foi submetida à osteossintese através datransfixação externa e gesso, associada com a aplicação intralesional deplasma rico em plaquetas (PRP). Após dois anos do tratamento, o animal iniciou programa de treinamento para corrida e, após seis meses do treinamento, correu o primeiro páreo oficial. A escolha dos métodos terapêuticos para o tratamento de fraturas em equinos deve ser baseada naquela que promova reparação precoce e de melhor qualidade, com menores riscos de complicações. Sendo assim, a associação terapêutica adotada foi considerada favorável, já que possibilitou o completo reestabelecimento da locomoção da paciente, podendo inclusive competir na sua modalidade esportiva.
\end{abstract}

Palavras-chave: equino, fratura, osteossintese, plasma rico em plaquetas.

\section{ABSTRACT}

In horses less than one year of age fractures of the third metacarpal bone (McIII) or metatarsal bone III (MtIII) are mainly attributed to trauma. Open reduction and internal fixation are the most common treatment method. A Quarter Horse filly with three months of age, which weighed $150 \mathrm{~kg}$ presented a diaphyseal multifragmentar wedge fracture of right MtIII which was treated with transcortical pins and cast, associated with intralesional application of platelet rich plasma (PRP). After two years of surgery, the animal initiated a training program for racing, and six months later, the patient ran its first official match. The choice of therapeutic methods for treating fractures in horses should be one that provides an earlier repair and minor possibility of complications. Thus, the therapy association which was adopted was considered favorable, since allowed full reestablishment of locomotion of the patient and made possible its return to race.

Key words: equine, fracture, osteosynthesis, platelet rich plasma.

Em potros até um ano de idade, as fraturas do III osso metacarpiano (McIII) ou III metatarsiano (MtIII) são principalmente atribuídas ao trauma (FITCH et al., 2001). Nas fraturas completas dos McIII/ MtIII, a redução aberta e a imobilização do foco com implantes constituem o tratamento cirúrgico recomendado (LEVINE \& RICHARDSON,2007). Porém, apesar da grande estabilidade proporcionada por esta técnica, esse tipo de fixação não proporciona o imediato e completo apoio de peso, pacientes apresentam moderada morbidade pós-operatória, risco de infecções e além da influência no processo de reparação (VAZ, 2006).

Sistemas de fixação externa são frequentemente utilizados em humanos e pequenos animais para promover a estabilização do foco da fratura sem invadi-lo. Em equinos, são utilizados para tratar fraturas de McIII, MtIII, multifragmentares, expostas ou com extensa lesão de tecidos moles (LESCUN et al., 2007).

\footnotetext{
'Departamento de Cirurgia e Anestesiologia Veterinária, Faculdade de Medicina Veterinária e Zootecnia, Universidade Estadual Paulista "Júlio de Mesquita Filho" (UNESP), Câmpus de Botucatu, Distrito de Rubião Jr, s/n, CP 560, 18618-970, Botucatu, SP, Brasil. E-mail: watanabe@fmvz.unesp.br.*Autor para correspondência.

"Médica Veterinária Autônoma, Brotas, SP, Brasil. 
O plasma rico em plaquetas (PRP) concentra uma grande variedade de fatores de crescimento e aumenta a taxa de proliferação de vários tipos celulares, além de desempenhar um papel importante na síntese e remodelamento do tecido de reparação (GRIFFIN et al., 2009; CARMONA et al., 2011). Assim, o objetivo desta nota é relatar o caso de uma potra com fratura diafisária multifragmentar em cunha de MtIII, tratada com pinos transcorticais e gesso, associados à injeção local de PRP.

Uma potra quarto de Milha com três meses de idade e $150 \mathrm{~kg}$ de peso foi encaminhada ao Hospital Veterinário - FMVZ - UNESP - Campus de Botucatu, com a queixa de impotência funcional do membro pélvico direito e desvio do eixo ósseo na região do MtIII, compatível com fratura completa e fechada. À anamnese, foi relatado que a potra encontrava-se solta em piquete com outros animais de idade similar e que a fratura foi observada durante a inspeção pela manhã.

Ao exame radiográfico, foi observada perda da continuidade óssea no terço médio do MtIII direito, fragmentos ósseos mediais ao foco de fratura, fratura distal dos MtII e MtIV, e discreto desvio lateral da região distal do MtIII (Figura 1A e 1C). Em virtude da característica multifragmentar em cunhada fratura, optou-se pela imobilização com pinos transversais, associada à imobilização externa com gesso sintético e aplicação local dePRP no foco de fratura.

Previamente à cirurgia, $100 \mathrm{~mL}$ de sangue venoso foram coletados, em tubos citrato de sódio a 3,8\%. O sangue coletado foi processado para a obtenção do PRP, conforme YAMADA (2011), através de centrifugação manual dupla e descarte de $50 \%$ do plasma sobrenadante, sendo utilizada a zona de névoa. A potra foi submetida à anestesia geral inalatória com isofluorano após ser pré-medicada com cloridrato de xilazina e induzida com cloridrato de quetamina. Durante a cirurgia, foram posicionados, transcorticalmente, quatro pinos de Steiman de 4,5mm de diâmetro por $300 \mathrm{~mm}$ de comprimento, sendo dois pinos proximais e dois distais ao foco de fratura. No peroperatório, 20mL de PRP, adicionados a $10 \%$ de cloreto de cálcio a $10 \%$ e $15 \%$ de trombina bovina, foram aplicados no foco da fratura. Uma bandagem Robert-Jones foi confeccionada do casco até o terço médio da tíbia e ataduras de gesso sintético foram distribuídas de maneira uniforme até o recobrimento total dos pinos.A medicação pós-operatória foi de ceftiofur (5mg. $\mathrm{kg}^{-1}$ IV SID, por 10 dias), flunixin meglumine (1,1mg.kg-1 IV SID, por 5 dias), 10.000 UI de soro antitetânico via subcutânea e omeprazol (4mg. $\mathrm{kg}^{-1}$ VO SID, por 40 dias).
$\mathrm{O}$ animal recebeu alta no $2^{\circ}$ dia pósoperatório (Figura 2A) sob os cuidados do médico veterinário da propriedade. Aos 40 dias de pósoperatório, foi realizado um exame radiográfico, no qual foi observado o início da formação do calo ósseo. Adicionalmente, foi observada intensa reabsorção óssea adjacente aos pinos proximais. Optou-se então pela retirada dos pinos e confecção de imobilização

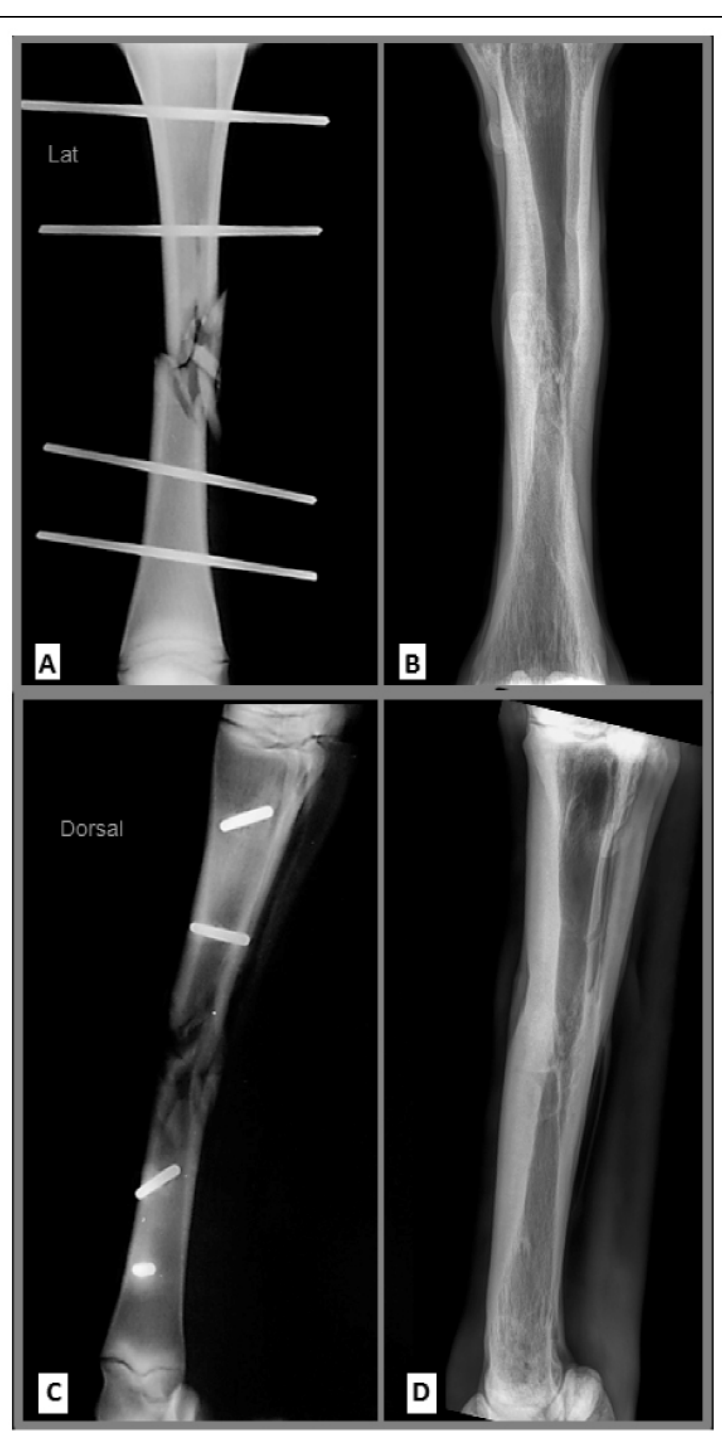

Figura 1 - Imagens radiográficas da região metatarsiana direita de equino fêmea da raça Quarto-de-Milha com 3 meses (A e C) e com 2 anos e 9 meses de idade (B e D). Nas projeções dorso plantar (A) e lateromedial (C): fratura multifragmentar em região média de MtIII e distal de MtII e MtIV, dois implantes metálicos transversais proximais e dois distais ao foco de fratura em região diafisária de MtIII. Nas projeções dorso plantar (B) e lateromedial (D): calo ósseo remodelado em região média de MtIII, com espessamento da cortical em região dorsal e lateral. 
externa com atadura gessada sintética. Na avaliação radiográfica aos 80 dias de pós-operatório, a formação do calo ósseo na região plantar era evidente, com grande preenchimento das linhas de fratura. A imobilização externa foi substituída por bandagem e tala de PVC por mais 10 dias.Durante todo o período, após a retirada da tala, a paciente foi acompanhada por meio de contato telefônico com informações de ausência de claudicação e a presença de discreto aumento de diâmetro do membro na região da fratura. Dois anos após a cirurgia, o animal iniciou um programa de treinamento para corrida similarmente aos outros animais (Figura 2B). Aos dois anos e seis meses do tratamento, novo exame radiográfico foi realizado observando-se remodelamento ósseo com espessamento da cortical (Figura 1B e 1D) e, desta forma, a paciente correu seu primeiro páreo (Figura 2C), terminando a prova de $5^{\circ}$ lugar entre seis participantes.

Nas fraturas completas do Mc/MtIII em equinos jovens, recomenda-se o tratamento cirúrgico, com redução aberta e imobilização do foco com duas placas de compressão dinâmica que visam à reconstrução anatômica do osso (BISCHOFBERGER et al., 2009; LEVINE \& RICHARDSON,2007). Porém, pela característica multifragmentar da fratura, optou-se pela imobilização com pinos transversais, sem a abordagem cirúrgica do foco, visando a minimizar lesões vasculares e aos tecidos moles adjacentes e diminuir o risco de contaminação bacteriana com consequente colonização do implante (LESCUN et al., 2007).

O processo de reparação de uma fratura é representado por eventos e mediadores biológicos, e pode ocorrer de maneira direta ou indireta, sendo que a ossificação direta é resultado do remodelamento ósseo por meio dos sistemas de Havers, em que as corticais ósseas buscam unir-se após a perfeita restauração anatômica dos fragmentos da fratura, como ocorre nos casos de fixação interna e aplicação de placas (VAZ, 2006). A indireta envolve a formação do tecido conjuntivo precursor, geralmente oriundo de um coágulo e depois de cartilagem, que prossegue com a calcificação intramembranosa e endocondral. Nesse caso, a cicatrização inicia-se com um processo inflamatório local, seguido de angiogênese e quimiotaxia de diversas moléculas e tipos celulares, finalizando com a formação de um calo ósseo competente (VAZ, 2006).

A cicatrização óssea indireta representa a principal forma fisiológica do processo de remodelação

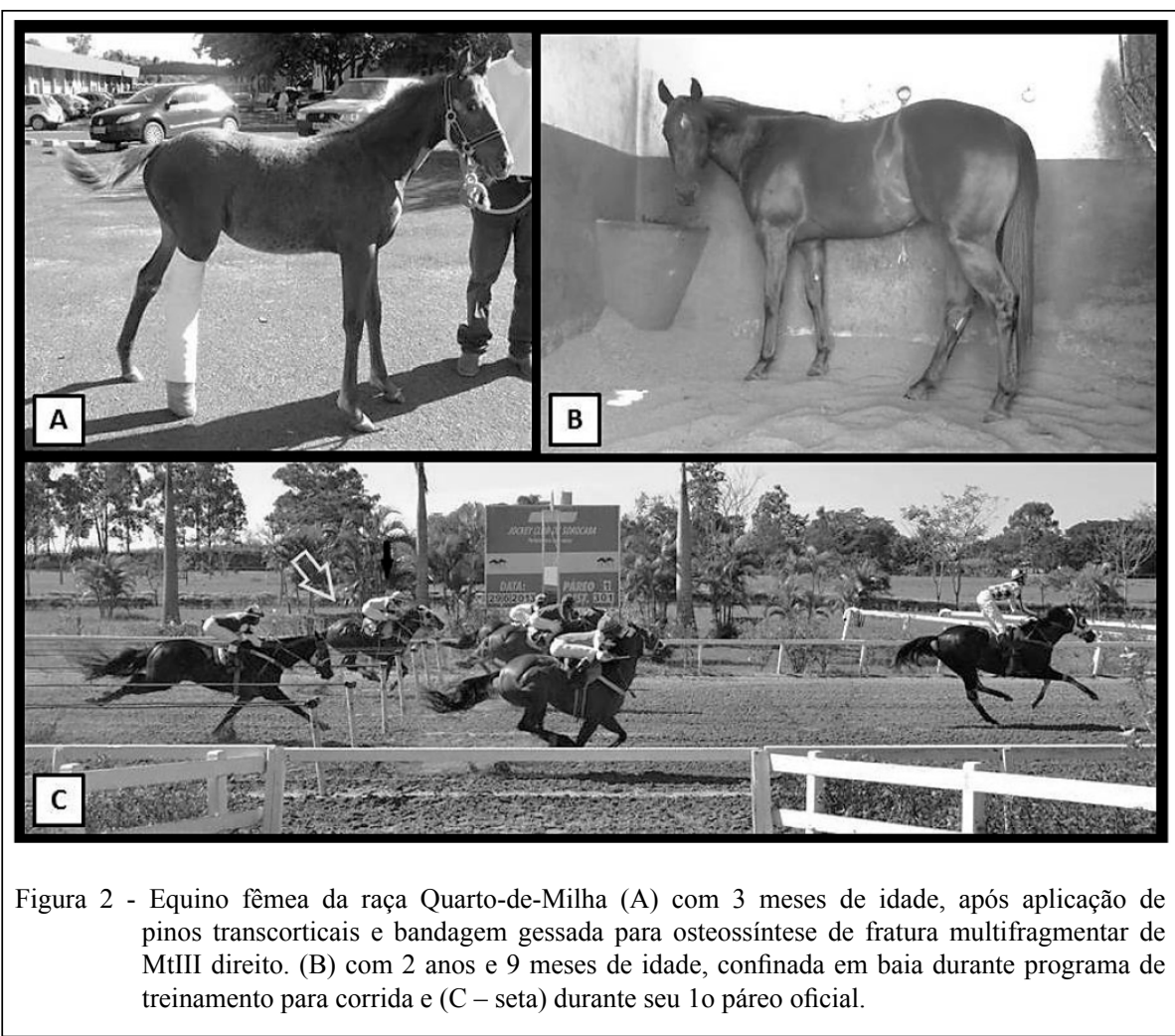

Ciência Rural, v.45, n.3, mar, 2015. 
na consolidação de uma fratura (JUKEMA et al., 1997). Sendo assim, no caso relatado, provavelmente a reparação foi indireta, pois fatores como redução da fratura com um alinhamento e imobilização perfeitos não foram alcançados (BISCHOFBERGER et al., 2009; VAZ, 2006).

Dentre as complicações relacionadas com a reparação das fraturas, a falha da união óssea decorrente da inadequada imobilização do foco de fratura (LOPES \&MARKEL, 2011) é condição preocupante nas fraturas de ossos longos em todas as espécies e está relacionada com grande morbidade e mortalidade na espécie equina (DUCHARME \& NIXON, 1996). No presente relato, a aplicação de pinos transcorticais incorporados ao gesso possibilitou imobilização suficiente do foco para permitir a formação do calo cartilaginoso, a formação das pontes entre os fragmentos e suas mineralizações (MARSELL \& EINHORN, 2011).

Apesar dos resultados contraditórios encontrados na literatura, inegavelmente o PRP possui uma alta concentração de fatores de crescimento, além de proteínas osteocondutoras, que estimulam a migração celular, a formação óssea e o incremento do tecido conjuntivo (GRIFFIN et al., 2009; BOSWELL et al., 2012). Observa-se uma influência positiva do PRP sobre a proliferação e celular, apresentando um significativo efeito quimiotáxico, mitogênico, angiogênico, analgésico e anti-inflamatório (BOSWELL et al., 2012), o que poderia sustentar a redução da morbidade dessa paciente e a formação de um calo ósseo consistente e precoce.

Ressalta-se que o osso é um dos poucos tecidos do organismo que mantém seu potencial de regeneração mesmo com a maturidade do indivíduo. Diferente de outros tecidos que cicatrizam com a formação de tecido fibroso com baixa qualidade, o osso se regenera e mantém suas propriedades mecânicas (GIANNOUDIS et al., 2007). Característica essa que possibilitou à paciente treinar e correr um páreo de maneira segura, mesmo com histórico de fratura completa do MtIII.

A escolha de métodos terapêuticos para o tratamento de fraturas em equinos deve ser baseada na possibilidade de reparação precoce e de qualidade, com menor risco de complicações. Nesse sentido, considerou-se favorável o método adotado, o que possibilitou que a paciente, além de ter um restabelecimento da locomoção, pudesse competir na sua modalidade esportiva.

\section{REFERENCIAS}

BISCHOFBERGER, A.S; FÜRST A. et al. Surgical management of complete diaphyseal third metacarpal and metatarsal bone fractures: Clinical outcome in 10 mature horses and 11 foals. Equine Veterinary Journal, v.41, n.5, p.465-473, 2009. Disponível em: <http://www.ncbi. nlm.nih.gov/pubmed/19642407>. Acesso em: 15 set. doi: 10.2746/042516409X389388.

BOSWELL, S.G.; COLE, B.J. et al. Platelet-rich plasma: a milieu of bioactive factors. Journal of Arthroscopic and Related Surgery, v.28, n.3, p.429-439, 2012. Disponível em: $\quad<$ http://orthodoc.aaos.org/WilliamFBennettMD/PRP\%20 Bioactive $\% 20$ Factors.pdf $>$. Acesso em: 15 set. doi:10.1016/j. arthro.2011.10.018

CARMONA, J.U.; LOPEZ, C. et al. Uso de concentrados autólogos de plaquetas como terapia regenerativa de enfermedades crónicas del aparato musculoesquelético equino. Archivos de Medicina Veterinaria, v.43, p.1-10, 2011. Disponível em: <http://www.scielo.cl/pdf/amv/v43n1/art02. pdf $>$. Acesso em: 12 out. 2012.

DUCHARME, N.G.; NIXON, A.J. Delayed union, nonunion and malunion. In: NIXON, A.J. Equine fracture repair. Philadelphia: Saunders 1996, Chapt.36, p.354358,1996 .

FITCH, G.L.; GALUPPO, L.D et al. An in vitro biomechanical investigation of an intramedullary nailing technique for repair of third metacarpal and metatarsal fractures in neonates and foals. Veterinary Surgery, v.30, p.422-431, 2001. Disponível em: <http://www.pubfacts.com/detail/11994848/An-in-vitrobiomechanical-investigation-of-an-MP35N-intramedullaryinterlocking-nail-system-for-repa $>$. Acesso em: 15 set. doi:10.1053/jvet.2001.25866.

GIANNOUDIS, P.V. et al. Fracture healing: the diamond concept. Injury International Journal Care Injured, v.38, suppl., p.S3-S6, 2007. Disponível em: <http://www.ortopaedi. $\mathrm{dk} /$ fileadmin/specialespecifikke_kurser/traumatologi/2013/ litteratur/diamond_concept.pdf $>$. Acesso em: 15 set. doi:10.1016/ S0020-1383(08)70003-2.

GRIFFIN, X.L.; SMITH, C.M. et al. The clinical use of plateletrich plasma in the promotion of bone healing: A systematic review. Injury International Journal Care Injured, v.40, p.158-162, 2009. Disponível em: <http://www.injuryjournal. com/article/S0020-1383(08)00277-5>. Acesso em: 15 set. doi:10.1016/j.injury.2008.06.025.

JUKEMA, G.N.; SETTNER, M. et al. High stability of the Ilizarov ringfixator in a metacarpal fracture of an Arabian foal. Archives of Orthopaedic and Trauma Surgery, v.116, n.5, p.287-289, 1997. Disponível em: <http://link.springer.com/ article/10.1007/BF00390055>. Acesso em: 15 set. doi: 10.1007/ BF00390055.

LESCUN, T.B.; MCCLURE, S.R. et al. Evaluation of transfixation casting for treatment of third metacarpal, third metatarsal, and phalangeal fractures in horses: 37 cases (1994-2004). Journal of the American Veterinary Medical Association, v.230, n.9, p.1340-1349, 2007. Disponível em: <http://www.ncbi.nlm. nih.gov/pubmed/17472561>. Acesso em: 15 set. doi: 10.2460/ javma.230.9.1340. 
LEVINE, D.G.; RICHARDSON, D.W. Clinical use of the locking compression plate (LCP) in horses: a retrospective study of 31 cases (2004-2006). Equine Veterinary Journal, v.39, n.5, p.401-406, 2007. Disponível em: <http://onlinelibrary.wiley. com/doi/10.2746/042516407X196555>. Acesso em: 15 set. doi: 10.2746/042516407X196555.

LOPES, M.J.; MARKEL, M.D. Chapter 74: Bone biology and fracture healing. In: AUER, J.; STICK, J. Equine Surgery. 4.ed. Philadelphia: Saunders, 2011. Chapt.34, p.1025-1040.

MARSELL, R.; EINHORN, T.A. The biology of fracture healing. Injury International Journal Care Injured, v.42, p.551-555,

2011. Disponível em: <http://www.injuryjournal.com/article/
S0020-1383(11)00125-2>. Acesso em: 15 set. doi: 10.1016/j. injury.2011.03.031.

VAZ, C.E.S. Avaliação do efeito de centrifugado osteogênico de medula óssea na consolidação de fratura. 2006. 97f. Tese (Doutorado em Ortopedia e Traumatologia) - Faculdade de Medicina da Universidade de São Paulo, SP.

YAMADA, A.L.M. Efeito do implante autólogo de plasma rico em plaquetas (PRP) e células tronco mesenquimais na reparação de lesões condrais articulares induzidas experimentalmente em equinos. 2011. 111f. Dissertação (Mestrado em Cirurgia Animal)Faculdade de Medicina Veterinária e Zootecnia, Universidade Estadual Paulista, Botucatu, São Paulo. 